\title{
The median recoil direction as a WIMP directional detection signal
}

\author{
Anne M. Green ${ }^{1}$ and Ben Morgan ${ }^{2}$ \\ ${ }^{1}$ School of Physics and Astronomy, University of Nottingham, University Park, Nottingham, NG7 2RD, UK \\ ${ }^{2}$ Department of Physics, University of Warwick, Coventry, CV4 7AL, UK
}

\begin{abstract}
Direct detection experiments have reached the sensitivity to detect dark matter WIMPs. Demonstrating that a putative signal is due to WIMPs, and not backgrounds, is a major challenge however. The direction dependence of the WIMP scattering rate provides a potential WIMP 'smoking gun'. If the WIMP distribution is predominantly smooth, the Galactic recoil distribution is peaked in the direction opposite to the direction of Solar motion. Previous studies have found that, for an ideal detector, of order 10 WIMP events would be sufficient to reject isotropy, and rule out an isotropic background. We examine how the median recoil direction could be used to confirm the WIMP origin of an anisotropic recoil signal. Specifically we determine the number of events required to confirm the direction of solar motion as the median inverse recoil direction at $95 \%$ confidence. We find that for zero background 31 events are required, a factor of $\sim 2$ more than are required to simply reject isotropy. We also investigate the effect of a non-zero isotropic background. As the background rate is increased the number of events required increases, initially fairly gradually and then more rapidly, once the signal becomes subdominant. We also discuss the effect of features in the speed distribution at large speeds, as found in recent high resolution simulations, on the median recoil direction.
\end{abstract}

PACS numbers: 95.35.+d

\section{INTRODUCTION}

Weakly Interacting Massive Particles (WIMPs), and in particular the lightest neutralino in supersymmetric models, are a well motivated dark matter candidate [13]. WIMPs can be detected directly, in the lab, via the elastic scattering of WIMPs on detector nuclei [4]. Experiments now have the sensitivity required to probe the theoretically favoured regions of parameter space [5-7] and the CDMS experiment has recently observed two events in its WIMP signal region [5].

Neutrons, from cosmic-ray induced muons or natural radioactivity, can produce nuclear recoils which (on an event by event basis) are indistinguishable from WIMP induced recoils. Furthermore perfect rejection of other backgrounds is impossible, for instance 'surface events' (electron recoils close to the detector surface) in the case of CDMS. As highlighted by the CDMS events, as well as the long-standing DAMA annual modulation signal [8], demonstrating the WIMP origin of a putative signal is absolutely crucial. The direction dependence of the WIMP scattering rate (due to the Earth's motion with respect to the Galactic rest frame) [9] provides a potential WIMP 'smoking gun'. Assuming that the WIMP distribution is predominantly smooth, the peak WIMP flux comes from the direction of solar motion (towards the constellation Cygnus) and the recoil rate is then peaked in the opposite direction. The recoil rate, in the Galactic rest frame, is highly anisotropic; the rate in the forward direction is roughly an order of magnitude larger than that in the backward direction. A detector capable of measuring the nuclear recoil vectors (including the sense $+\mathbf{p}$ versus $-\mathbf{p}$ ) in 3-dimensions, with good angular resolution, could reject isotropy of the recoils with only of order 10 events [10, 11]. Most, but not all, backgrounds would produce an isotropic Galactic recoil distribution (due to the complicated motion of the Earth with respect to the Galactic rest frame). An anisotropic Galactic recoil distribution would therefore provide strong, but not conclusive, evidence for a Galactic origin of the recoils.

Confirmation of the WIMP origin could be obtained by verifying that the properties of the anisotropy match the expectations for WIMP induced recoils. Assuming the WIMP distribution is dominated by a smooth component, the median inverse recoil direction should be compatible with the direction of solar motion.

To summarise, a WIMP search strategy with a directional detector could be divided into a sequence of phases: 1. Search phase (detection of non-zero recoil signal)

2. Detection of anisotropy

3. Study of properties of anisotropy

which require successively larger numbers of events (and hence larger exposures).

The initial simple search phase aims to detect an anomalous recoil signal above that expected from backgrounds. To claim an anomalous signal inconsistent with zero at $95 \%$ confidence requires $4-5$ events. The second step, as discussed above, would be to check whether the Galactic recoil directions are anisotropic and would require of order 10 events (assuming zero background). In this paper we focus on the third phase, examining how measuring the median recoil direction could be used to provide confirmation of the WIMP origin of an anisotropic recoil signal. In section II we describe the input to our Monte Carlo simulations. In section [II we present our results, before concluding with discussion in Sec. IV 


\section{MODELLING}

We use the same statistical techniques and methods for calculating the directional nuclear recoil spectrum as in Refs. 11 13]. We briefly summarise these procedures here, for further details see these references and Ref. [14].

\section{A. Detector}

Many of the directional detectors currently under development 15, 16 are low pressure gas time projection chambers (TPCs), e.g. DMTPC [17], DRIFT [18, 19] and NEWAGE 20]. We therefore simulate a fairly generic TPC based detector. We assume that the the recoil directions, including their senses, are reconstructed perfectly in 3d. These are optimistic assumptions, therefore our results provide a lower limit on the number of events/exposure required by a real TPC based detector. For concreteness we use a $S$ target with an energy threshold of $20 \mathrm{keV}$. Finite angular resolution does not significantly affect the number of events required to detect anisotropy [11, 21], provided it is not worse than of order $10^{\circ}$. Axial and/or 2-d read-out would, however, significantly degrade the detector capability [11 13, 21].

\section{B. WIMP properties and distribution}

The detailed angular dependence of the recoil rate depends on the exact form of the WIMP velocity distribution [10, 11, 22]. However, if the WIMP velocity distribution is dominated by a smooth component the main features of the recoil distribution (rear-front asymmetry, median direction opposite to the direction of solar motion) are robust (see e.g. Ref. [11])). For concreteness we use the standard halo model halo, an isotropic sphere with local density $\rho=0.3 \mathrm{GeV} \mathrm{cm}^{-3}$ and a Maxwellian/gaussian velocity distribution with three dimensional velocity dispersion $\sigma_{\mathrm{v}}=270 \mathrm{~km} \mathrm{~s}^{-1}$, and fix the WIMP mass at $m_{\chi}=100 \mathrm{GeV}$.

Numerical simulations find velocity distributions with stochastic features at large speeds [23, 24]. Kuhlen et. al. [24] find that for high speed WIMPs $\left(v>500 \mathrm{~km} \mathrm{~s}^{-1}\right)$ the direction in which the WIMP flux is largest can deviate by more than $10^{\circ}$ from the direction of solar motion. The effect on the median recoil direction will be substantially smaller than this however. Firstly the setup we are considering $(S$ target with energy threshold of $20 \mathrm{keV}$ and $m_{\chi}=100 \mathrm{GeV}$ ) is sensitive to WIMPs with $v>225 \mathrm{kms}^{-1}$. Therefore a high speed feature will only contribute a small fraction of the WIMP flux. This will be true in general unless the WIMP mass is small and/or the threshold energy is large. Secondly, see e.g. fig. 3 of Ref. [11], due to the elastic scattering the recoil rate is less anisotropic than the WIMP flux. The deviation of the inverse median direction from the direction of solar motion will therefore be substantially smaller than the deviation of the peak WIMP flux. It will also depend on the (a priori unknown) velocity and density of the feature. For instance, for a stream with velocity, in Galactic coordinates, $\mathbf{v}_{\text {str }}=(-65,135,-249) \mathrm{km} \mathrm{s}^{-1}$ the difference between the inverse median direction and the solar direction only exceeds $5^{\circ}$ if the fraction of the local density in the stream exceeds $10 \%$ [11].

Since the effect of a feature in the speed distribution on the median recoil direction is expected to be small we do not investigate it in this study. If, with a large number of events, a statistically significant deviation of the inverse median direction were found, its origin could be investigated by studying the energy dependence of the deviation. We defer an investigation of this to future work.

Finally, direct detection experiments probe the ultralocal dark matter distribution on scales many orders of magnitude smaller than those resolved by simulations. It is not (and may never be) possible to directly calculate, or otherwise measure, the dark matter distribution on the relevant scales. In this case if WIMPs are detected, then the directional recoil rate could be used to reconstruct the ultra-local dark matter distribution [25].

\section{RESULTS}

The recoil rate is peaked in the direction opposite to the direction of solar motion. To allow ease of comparison with the direction of solar motion we use the inverse recoil directions (i.e. the directions from which the recoils originate) in our analyses.

We first examine how the accuracy with which the median Galactic recoil direction is determined depends on the number of WIMP events. The median direction is defined as the direction $\mathbf{x}_{\text {med }}$ which minimises the sum of the arclengths between $\mathbf{x}_{\text {med }}$ and the individual inverse recoil directions $\mathbf{x}_{\mathrm{i}}[26]$. It is found by minimising

$$
\mathcal{M}=\sum_{i=1}^{N} \cos ^{-1}\left(\mathbf{x}_{\text {med }} \cdot \mathbf{x}_{\mathrm{i}}\right),
$$

where $N$ is the number of events.

For a given number of WIMP events, $N_{\text {wimp }}$, we simulate $10^{4}$ experiments and in each determine the direction, $\mathbf{x}_{\text {med }}$, by minimizing Eq. (1) and hence calculate $\Delta$,

$$
\Delta=\cos ^{-1}\left(\mathbf{x}_{\text {med }} \cdot \mathbf{x}_{\odot}\right),
$$

the angle between the median direction and the direction of solar motion, $\mathbf{x}_{\odot}$. In Fig. 1 we plot the $50 \%$ and $95 \%$ percentiles of the distribution of $\Delta$ as a function of $N_{\text {wimp. }}$. We also investigate the effects of non-zero (isotropic) backgrounds. We parametrize the background rate in terms of the fraction of events which are signal, $\lambda=S /(S+B)$ where $S$ and $B$ are the signal and background rates respectively (c.f. Ref. [27]). For comparison we also plot the $5 \%$ and $50 \%$ percentiles for an purely isotropic distribution. 


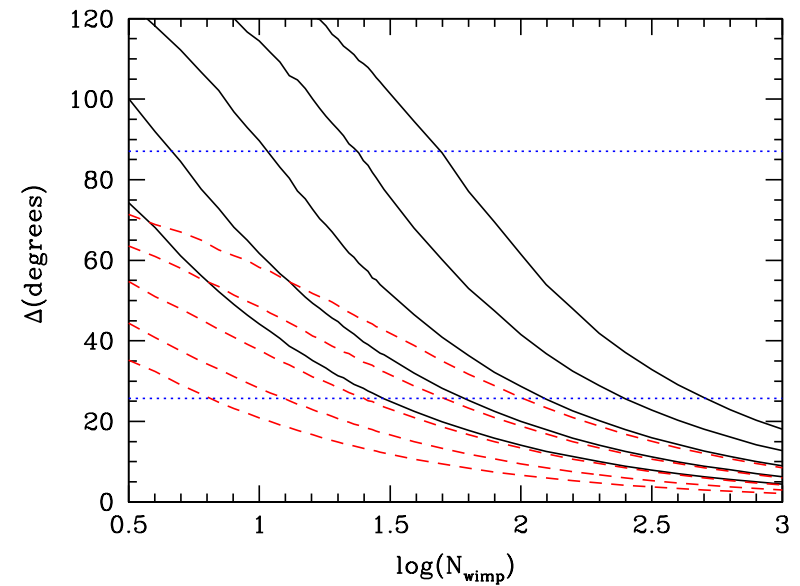

FIG. 1: The distribution of the angle between the median inverse recoil direction and the direction of solar motion, $\Delta$, as a function of the number of WIMP events, $N_{\text {wimp }}$, for varying signal fractions (from top to bottom) $\lambda=0.0625,0.125,0.25,0.5$ and 1 . The solid and dashed lines are the $95 \%$ and $50 \%$ percentiles. The dotted lines are (from top to bottom) the $50 \%$ and $5 \%$ percentiles for an isotropic recoil distribution.

We now determine the number of events required to confirm the direction of solar motion as the median inverse recoil direction at $95 \%$ confidence. We do this using the same methodology as in Ref. [11]. Briefly, we use the distribution of $\Delta$ for WIMP induced recoils and for the null hypothesis of isotropic recoils, to calculate the rejection and acceptance factors, $R$ and $A$. The rejection factor gives the confidence level with which the null hypothesis can be rejected given a particular value of the test statistic, while the acceptance factor is the probability of measuring a larger value of the test statistic if the alternative hypothesis is true, We then find the number of WIMP events required to give $A=R=0.95$ i.e. to reject the median direction being random at $95 \%$ confidence in $95 \%$ of experiments. This is shown in Fig. 2, as a function of the signal fraction $\lambda$. For zero background (i.e. $\lambda=1$ ) 31 events are required, a factor of $\sim 2$ more than are required to simply reject isotropy. The number of events required increases as $\lambda$ is decreased, initially fairly gradually and then, once the signal becomes subdominant $(\lambda \lesssim 0.5)$, more rapidly.

Billard et al. 27] have recently investigated using a sky-map based likelihood analysis to probe the correlation of the directional recoil rate with the direction of solar motion (and hence confirm the WIMP origin of a signal). Their results are qualitatively consistent with ours. For instance they find that, for a signal fraction $\lambda=0.5$, the peak signal direction can be confirmed to be within $20^{\circ}$ of the direction of solar motion with as few as 25 WIMP events. The median direction method is however faster and more robust. It particular it has the advantage of being model independent (i.e. one does

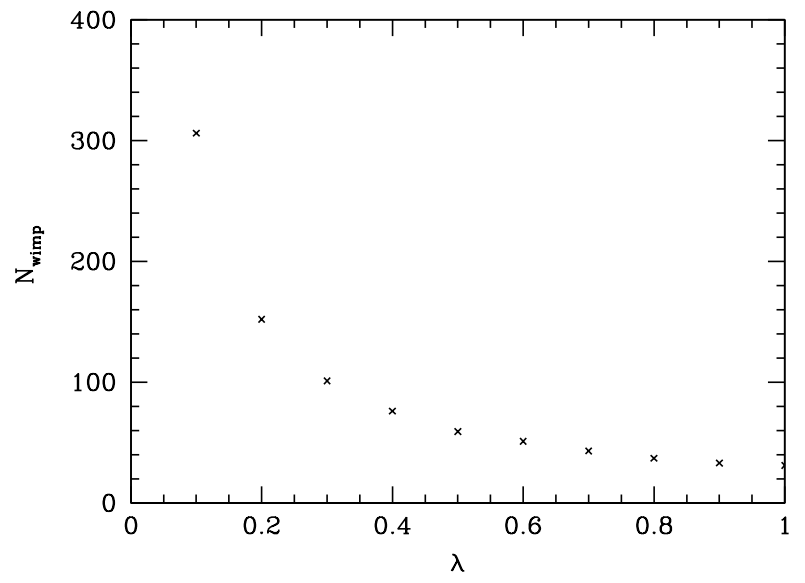

FIG. 2: The dependence of the number of WIMP events, $N_{\text {wimp }}$, required to reject the median direction being random at $95 \%$ confidence in $95 \%$ of experiments on the signal fraction, $\lambda$.

not need to assume an exact form of the WIMP velocity distribution).

If desired, further confirmation of the WIMP origin of an anisotropic recoil signal could be obtained by studying the variation of the median recoil direction in the lab frame [18]. Over the course of a sidereal day the peak in the recoil distribution traces out a small circle on the sky. For a detector located in the Northern hemisphere during a sidereal day the peak recoil direction in the lab rotates (roughly) from down to South and back. A periodogram analysis could be used to verify that median direction varies over a sidereal, rather than solar, day (c.f. Ref. 28] for the annual modulation).

\section{DISCUSSION}

In this paper we have investigated how the median recoil direction in directional detection experiments can be used as a WIMP signal. Assuming a smooth WIMP distribution, the peak WIMP flux is from the direction of solar motion and the median recoil direction is in the opposite direction. An ideal detector could reject isotropy of recoils with only of order 10 events 10,11$]$. Confirmation of the WIMP origin of an anisotropic recoil signal could be obtained by studying the details of the anisotropy and in particularly confirming that the median inverse Galactic recoil direction coincides with the direction of Solar motion. We find that, with an ideal detector and zero-background, to confirm the direction of solar motion as the median inverse recoil direction at 95\% confidence requires 31 events (see also Ref. [27]). Non-zero isotropic background would increase this number, significantly if the signal is sub-dominant.

For concreteness (and in the absence of a definitive alternative) we have used the standard halo model halo, 
which has an isotropic Maxwellian speed distribution. While the detailed angular dependence of the recoil rate depends on the exact form of the WIMP velocity distribution, if the WIMP velocity distribution is dominated by a smooth component the median inverse recoil distribution will be close to the direction of solar motion (see e.g. Ref. [11]). Recent high resolution simulations have found stochastic, features in the speed distribution at large speeds 23, 24]. The direction in which the flux of high speed WIMPs is largest can deviate by more than $10^{\circ}$ from the direction of solar motion [24]. However the deviation of the median inverse direction will be small compared with that expected from an isotropic recoil di- rection. We therefore conclude that features in the speed distribution at high speed are unlikely to affect the utility of the median recoil direction as a WIMP signal. If, with a large number of events, a statistically significant deviation of the inverse median direction were found, its origin could then be investigated by studying the energy dependence of the deviation.

\section{Acknowledgments}

AMG and BM are supported by STFC.
[1] G. Jungman, M. Kamionkowski and K. Griest, Phys. Rep. 267, 195 (1996).

[2] G. Bertone, D. Hooper and J. Silk, Phys. Rep. 405, 279 (2005).

[3] Particle Dark Matter, observations, models and searches, ed. G. Bertone, CUP (2010).

[4] M. W. Goodman and E. Witten, Phys. Rev. D 31, 3059 (1985).

[5] Z. Ahmed et al., arXiv:0912.3592.

[6] J. Angle et al., Phys. Rev. Lett. 100, 021303 (2008) arXiv:0706.0039.

[7] V. N. Lebedenko, Phys. Rev. D 80, 052010 (2009) arXiv:0812.1150.

[8] R. Bernabei, Eur. Phys. C 56, 333-355 (2008) arXiv:0804.2741

[9] D. N. Spergel, Phys. Rev. D 37, 1353 (1988).

[10] C. J. Copi, J. Heo and L. M. Krauss, Phys. Lett. B 461, 43 (1999) astro-ph/990449; C. J. Copi and L. M. Krauss, Phys. Rev. D 63, 043507 (2001) astro-ph/0009467.

[11] B. Morgan, A. M. Green and N. J. C. Spooner, Phys. Rev. D 71103507 (2005) astro-ph/0408047.

[12] B. Morgan and A. M. Green, Phys. Rev. D 72, 123501 (2005) astro-ph/0508134.

[13] A. M. Green and B. Morgan, JCAP08(2007)022 astro-ph/0609115

[14] B. Morgan, Dark Matter Detection With Gas Time Projection Chambers, Ph.D. Thesis, University of Sheffield, 2004 .
[15] G. Sciolla and C. J. Martoff, New J. Phys. 11105018 (2009) arXiv:0905.3675.

[16] S. Ahlen et al. Int. J. Mod. Phys. A 25 1-51 (2010) arXiv:0911.0323

[17] G. Sciolla et al., J. Phys. Conf. Ser. 179012009 (2009) arXiv:0903.3895

[18] D. P. Snowden-Ifft, C. J. Martoff, and J. M. Burwell, Phys. Rev. D 61, 1 (2000) astro-ph/9904064

[19] G. J. Alner et al., Nucl. Inst. and Meth. A. 535, 644 (2004).

[20] T. Tanimori et al., Phys. Lett. B 578, 241 (2004) astro-ph/0310638

[21] C. J. Copi, L. M. Krauss, D. Simmons-Duffin and S. R. Stroiney, Phys. Rev. D 75, 023614 (2007) astro-ph/0508649

[22] M. S. Alenazi and P. Gondolo, Phys. Rev. D 77043532 (2008) arXiv:0712.0053.

[23] M. Vogelsberger et al., Mon. Not. Roy. Astron. Soc. 395, 797 (2009) arXiv:0812.0362.

[24] M. Kuhlen et al. arXiv:0912.2358.

[25] P. Gondolo, Phys. Rev. D 66, 103513 (2002) hep-ph/0209110

[26] N. I. Fisher, T. Lewis and B. J. J. Embleton, Statistical analysis of spherical data, CUP, (1987).

[27] J. Billard, arXiv:0911.4086.

[28] Y. Ramachers, Astropart. Phys. 19, 419 (2003). 